\title{
A universal, damage-criterion-independent cycle counting method for multiaxial and variable amplitude fatigue
}

\author{
K. G. F. Janssens ${ }^{1, *}$ \\ ${ }^{1}$ Laboratory for Nuclear Materials, Nuclear Energy and Safety Research Division, Paul Scherrer Insti- \\ tute, Forschungsstrasse 111, 5232 Villigen PSI, Switzerland
}

\begin{abstract}
None of the procedures for cycle-counting defined in the ASTM document with designation E1049-85 (Reapproved 2017) [1] are generally applicable to non-proportional, multi-axial Fatigue. In addition, as the concepts of amplitude and mean stress are defined per cycle, their values are dependent (or co-define) the cycle counting method. This poses an obvious problem to the analysis of non-proportional, multi-axial fatigue damage, as lifetime is, not in all but in many cases, an amplitude and mean stress dependent material property. Most of the newer cycle counting methods developed till date are at least inspired by the works of Wang \& Brown [2] and of Bannantine \& Socie [3], both of which are themselves still frequently used. Being built inspired by counting methods developed for uniaxial cycling, all of the approaches to date known to this author are limited in a way that is very well phrased by Anes et al [4], whom, on page 79 of their article, write that (quote): The damage criterion is the base stone to set up random fatigue. The damage parameter must capture the fatigue damage behavior to allow set up a cycle counting method and an accumulation model. Challenging this statement, a new cycle counting procedure is presented that is completely independent of the damage criterion, and universally works from the simplest uniaxial experiment, to the most complex, variable amplitude and frequency, non-proportional multiaxial fatigue loading. The definition of this new cycle counting concept is surprisingly simple. Despite of its simplicity, the new cycle counting procedure has different advantages when compared to the procedures known to date. Its standalone definition, allows it to be combined with any damage criterion. It does not require periodicity of the loading cycle, and can therefore be straightforwardly used to analyze variable frequency and amplitude, multiaxial fatigue loading.
\end{abstract}

\section{Introduction}

The ASTM document with designation E1049-85 (Reapproved 2017) [1] compiles a list of acceptable procedures for cycle-counting methods employed in fatigue analysis. The list includes the counting methods level-crossing, peak-to-peak, simple-range, and specifically for variable amplitude loading the rainflow counting and related methods. None of these methods are generally applicable to the non-proportional, multi-axial loading case. The reason for this is that there is no universal way to project the (cycling) stress or strain tensor on a scalar value without the loss of information relevant to the cyclic damage evolution in the

\footnotetext{
*e-mail: koen.janssens@psi.ch
} 
microstructure of the material. In addition, as the concepts of amplitude and mean stress are defined per cycle, their values are dependent on the cycle counting method. This poses an obvious problem to the analysis of non-proportional, multi-axial fatigue damage, as lifetime is an amplitude and mean stress dependent material property.

This is not a new problem and has been addressed using different approaches by different research groups. Carpinteri et al [5] use a spectral approach that has a focus on random non-proportional multiaxial loading. Their method consists of a critical plane method, the stresses on which are analyzed using a frequency-domain criterion. The same research group [6] recently reviewed some of the existing criteria for multiaxial fatigue analysis for random variable amplitude loading. Wang and Susmel [7] report on the performance of their (critical-plane-based) modified Manson-Coffin curve method in a context of variable amplitude multiaxial load histories, the latter for which the authors determine the orientation of the critical plane through that direction along which the variance of the resolved shear strain reaches it maximum value. Under variable amplitude complex load histories, this direction was also used to count the resolved shear strain cycles via the classic Rain-Flow method. One should take notice that both the methods of Carpinteri and Susmel use a fixed orientation for the critical plane, albeit determined using different procedures, thereby potentially losing part of the cyclic loading data needed to count cycles.

This way of defining criteria is commonly used, also when observing crack propagation. For example, Mei et al [8] present an approach in which one characterizes the multiaxial loading cycle by computing a critical plane based on stress intensity factors of different modes. In [9] the same research group presents a case study applying their approach to welded plates. The paper of Gates and Fatemi [10] evaluates the methodology for, and effectiveness of, common equivalent stress- and strain- based fatigue life analysis approaches when applied to variable amplitude multiaxial service loading conditions. The authors find mixed results, especially for un-notched specimens, where multiaxial loading effects come into play, and no approach considered is able to predict more than $60 \%$ of fatigue data within a factor of \pm 3 of experimental life. More importantly, they observe a consistent trend of non-conservative life predictions for variable amplitude loading conditions. Concerning cycle counting, these authors write that (quote): For non-proportional loading, however, ... there may be no clear definition of a cycle. Therefore, the amplitudes and mean stresses of the different loading components, required as input for the equivalent stress/strain approaches, can change depending on the component used for cycle counting ... making the application of these approaches vague under such conditions. The authors also list a number of ways to deal with this problem, but conclude that none of these are without drawbacks. Herewith Gates and Fatemi point to the problem treated in this article, and write that (quote): Despite these shortcomings, equivalent stress/strain based fatigue life analyses are still commonly used in situations involving multiaxial variable amplitude loadings. This is due, in part, to the simplicity of such approaches, as well as to the lack of well-established alternative procedures. Therefore, being able to assess the accuracy of equivalent stress/strain methods, when applied to complex fatigue life analysis problems, is a topic of interest for many practical situations.

Meggiolaro et al [11] address an aspect of cycle counting that is oft overseen, more precisely the fact that damage modeling, when based on cycle counting, is usually a form of post-processing that can only compute the accumulated fatigue damage after a full cycle or half cycle has been identified. This stands in contrast to the true nature of damage accumulation, which is a strain-increment-based process, and not a cycle based one. Meggiolaro et al present a damage accumulation model that functions without cycle counting, but instead uses a type of approach inspired by continuum damage mechanics theory. The authors start by evaluating two variants, namely an elasto-plastic energy integration approach, and a so-called incremental fatigue damage approach. The energy integration approach has the 
limitation that it makes use of the plastic strain, disqualifying it for most high cycle fatigue conditions. In addition, in their evaluation of this approach, the authors point out that some of its parameters must be cycle dependent after all. Ultimately the authors propose a further development of an incremental fatigue damage approach, which is strongly dependent on the description of the (cyclic) plastic hardening behavior of the material.

\section{A universal cycle counting method}

Conventionally, cycles are counted peak-to-peak, the details are in how these peaks are selected to count. An alternative approach, which is proposed here for the first time, is to use the quantifiable measure of the continuous change of direction in the stress or strain path. The concept can be used in any stress or strain space, including those of higher dimensions, but in this article only the 2-dimensional stress space, as it exists on the free surface of a specimen, is considered. An important aspect of the new approach, is that it is unrelated and therefore completely independent of the choice of damage criterion used with it. This puts it in contrast to the many, popular critical-plane-criteria-based counting methods available in literature.

When counting cycles for uniaxial or proportional multiaxial loading using the conventional peak-to-peak approach, the loading path is a linear one in stress space, and one can straightforwardly count the number of reversals occurring in the path direction, two reversals adding up to a complete cycle in the simplest case. The amplitude is then half the range between those reversal points; the mean stress the average of the values observed at those 2 points. In figure 1(a) and (b) this is illustrated for a uniaxial and a proportional, multiaxial path. In the non-proportional loading case this can indeed be tricky, e.g. in figure 1(c) a path is shown in which no unique stress or strain path reversal point can be determined, because the horizontal and vertical components are out of phase in time. One can solve this by, for example, evaluating the reversals in each component independently and post-process the observations to combine them, e.g. using a critical plane rainflow based approach as described in [12]. In figure 1(d) a loading path is shown for which cycle counting may become somewhat ambiguous: either the complete path is considered as a single cycle, in which case the amplitude definition can be a challenge, or either it is considered to be a sequence of two different cyclic paths (left and right of the starting point on the vertical axis) in alternating repetition, albeit with an alternating mean stress. Considering variations of the path in figure 1(d) in which many smaller cycles constitute a single larger cycle, one can easily understand that this can be a problem in the analysis of non-proportional fatigue. This loading case is not uncommon when a main cycle is perturbed by higher frequency and lower amplitude cycling [13].

An alternative, new method to count cycles is now proposed, which can be used in both proportional and non-proportional loading cases, and which respects the conventional, peakto-peak cycle counting method for the case of proportional cycling. The approach is defined by measuring the directional change of the stress or strain vector in its tensorial space, which can be explained intuitively in a two-dimensional space for the paths plotted in figure 1 . In the proportional cases (a) and (b), the path vector makes a $180^{\circ}$ turn every time it ends a half-cycle. This means that a half cycle is defined by a $180^{\circ}$ orientation change. The same orientation-change principle can be applied incrementally, without resulting in a change of the counted number of cycles for the proportional conditions. For the non-proportional path conditions in figure 1(c) and (d), one can count the cycles by keeping track of the accumulated orientation change. For figure 1 (c) this adds up to $180^{\circ}$ per half-circle or 1 cycle per circle. In the path shown in figure $1(\mathrm{~d})$ one counts six $90^{\circ}$ corners, adding up to $540^{\circ}$ total orientation change or one and a half cycle per completion of the plotted pattern. Should corners occur 

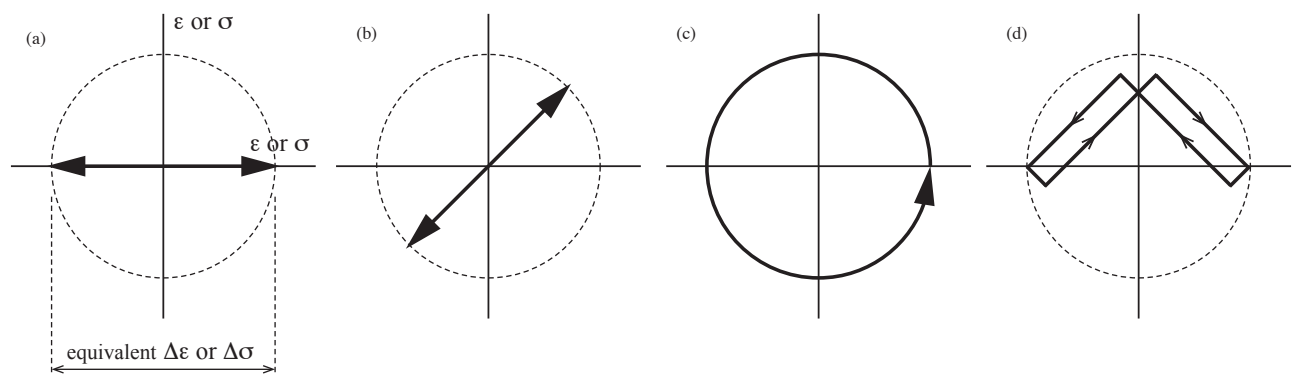

Figure 1. Cycles plotted in two-dimensional, principal stress or strain space, with exactly the same amplitude according to the definition proposed by Jiang in [14]. This definition is still commonly used in engineering standard codes.

in the path, of which the angles add up to a total not equal to $180^{\circ}$, one needs to split up the corner to assure half cycles are exactly $180^{\circ}$. One can argue that this must be so, as in reality corners are always smooth when observing from sufficiently close-up.

Despite of its simplicity, this new cycle counting procedure has different advantages when compared to the procedures known to date. Its standalone definition, allows it to be combined with any damage criterion. It does not require periodicity of the loading cycle, and can therefore be straightforwardly used to analyze variable frequency and amplitude loading.

\section{Illustrations of the method applied to different cycling paths}

In this section examples are given illustrating the new cycle counting approach, as defined in the previous section. Figure 2 shows 2 examples of multiaxial paths: on the left a nearproportional one, on the right a non-proportional one consisting of two $90^{\circ}$ out-of-phase sinusoidal paths. The frequency is chosen to return one loading block per arbitrary unit of time, which allows the new counting method to be directly compared to the visually-observed number of cycles; the bottom row of plots illustrates the method functions as expected for these simple cases, in that one loading block is indeed counted as one cycle.

In figure 3 an example is presented of a more complex cyclic path. In the figure the left column shows (a) the path in stress or strain space, (b) the same against time using unit of time per conventional, visual cycle, and finally (c) the primary cycles counted using the new method versus time. In the column on the right the same procedure is used to analyze what I propose to name secondary cycling, which will be explained in more detail in the next section.

\section{Second order path and cycle counting}

When the new counting method is used on a cyclic path, the point in stress or strain space at which a cycle is counted and the next cycle begins, can vary from cycle to cycle. Connecting these points in time constitutes a secondary path, which, if it turns out to be cyclic itself, can be analyzed using the exact same procedure. In essence, this secondary path returns a magnitude that is an approximation of the mean value of the cycles as classically defined. For the examples shown in figure 2 its value remains constant and equal to zero over time, once the point in space is reached where the first full cycle is counted. For example, assume a $90^{\circ}$ 


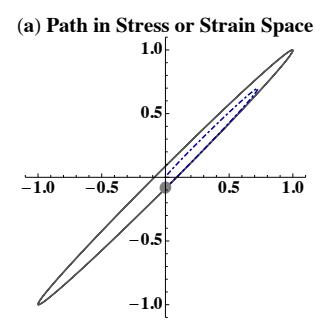

(c) Horizontal Component versus Time

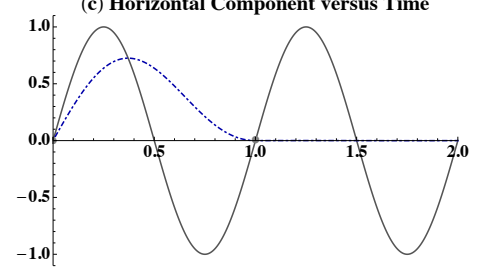

(e) Cycles Counted versus Time

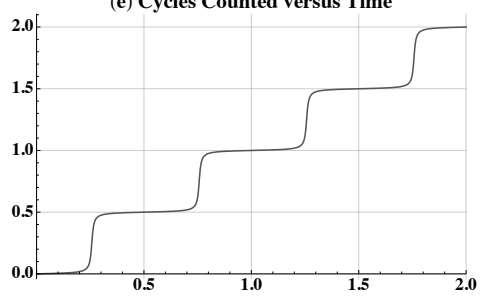

(b) Path in Stress or Strain Space

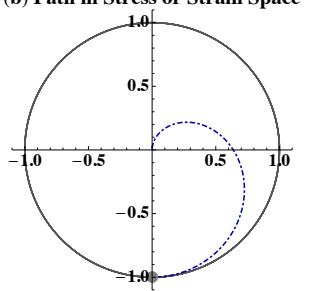

(d) Horizontal Component versus Time

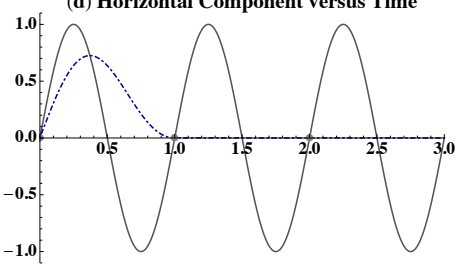

(f) Cycles Counted versus Time

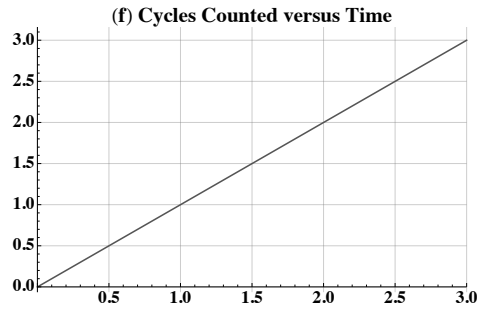

Figure 2. Example of near-proportional and a non-proportional biaxial cycle in stress or strain space. Arbitrary units on the axes are used. The dashed blue line is the per counted cycle average position in path space.

out-of-phase stress path, as shown on the right in figure 2, is started from the loading to stress $(0,-1)$, and continued by cycling counter-clock-wise. Every loading block, i.e. one circle, a total path orientation change equal to $360^{\circ}$ is registered at this same point $(0,-1)$, marked with a gray dot in the figures.

In figure 3, the same procedure is followed for a more complex loading block. The path in stress space is plotted in 3(a) in gray, the vertical and horizontal components versus time in 3(c) and (d); always using a gray dot to mark the position at which a cycle is counted. The dashed line in figure 3(b) connects these points to give the reader some insight into the sequence of where completed cycles are observed. In parts (a), (b), (c) and (d), the blue, dotdashed line plots the average position observed over the past cycle at the given point in time. To compute this average at any point on the path, the path is traced back from the current point until to the point from which the accumulated path orientation change at the current point is observed equal to $360^{\circ}$. The average position at the current point is then computed from this, traced-back section of the path; except for the values plotted before the first full cycle is counted, as there are no data points before time zero.

The path constituted by following this procedure is hereby named a second order path in loading space, be it stress or strain.

Figure 3 is a good example of how this second order path can be interpreted. In this example both the vertical and the horizontal loading blocks consist of a superposition of a lower and a higher frequency sinusoidal curve. Where the first order cycles counted concern the 


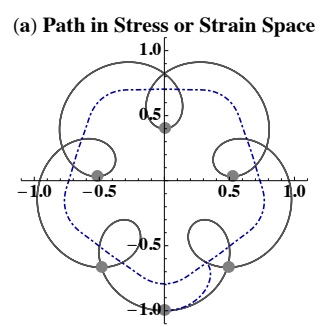

(c) Horizontal Component versus Time
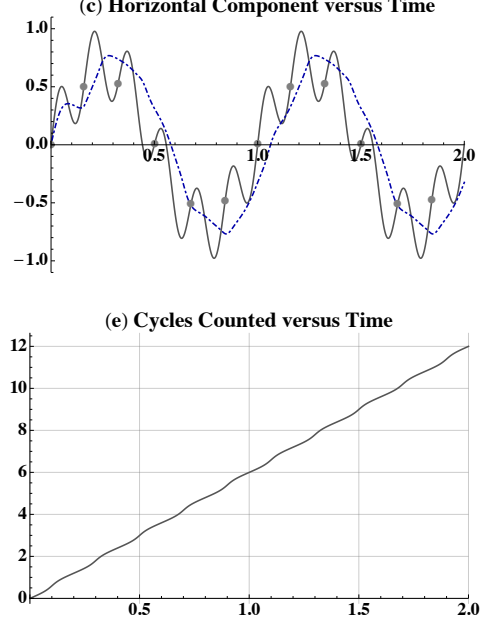

(b) $2^{\text {nd }}$ Order Path in Stress or Strain Space

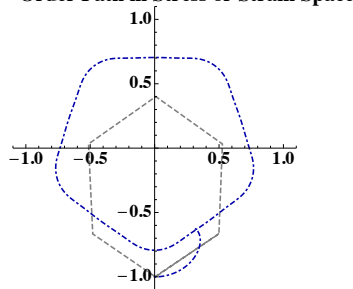

(d) Vertical Component versus Time
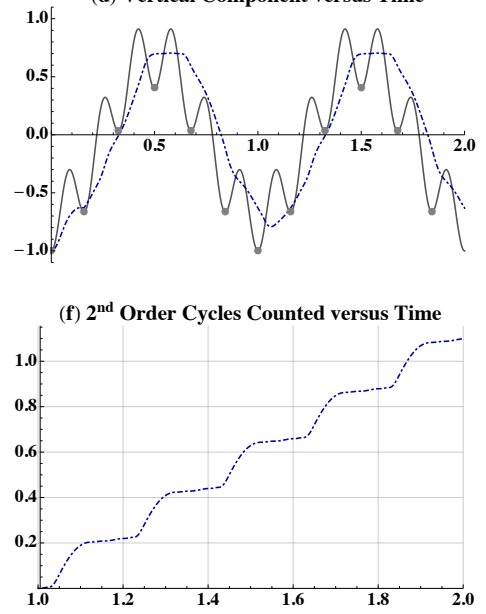

Figure 3. Example non-proportional biaxial cycle. Arbitrary units on the axes are used. The definition of a $2^{\text {nd }}$ order path is given in the text.

higher frequency component, the second order cycles counted concern the component with the lower frequency. This is expected to be a very useful feature of the method when analyzing variable amplitude multiaxial cycling, and for the case when the mean value shifts with time and ratcheting is involved in the loading pattern. For example, one could opt to count only the first order cycles, while observing the mean stress at each cycle counted, and letting that information flow into a Mines-rule damage accumulation criterion. Or, alternatively, one could opt to count the first order cycles without observing their cyclic mean values, but instead also count the second order cycles, and combine first and second order in a Mines-rule damage accumulation criterion. The number of second order cycles counted is plotted in part (f) of the figure. In the path shown in figure 3 one thus counts 6 first order and roughly 1 second order cycles per loading block.

\section{Time, path or orientation based cycle averaging}

While the new, path-orientation-change-based cycle counting method defines a unique cycle, it has a peculiar aspect that becomes clear when one computes a cycle-derived property, such as the mean value of a cycle. Because the counting method solely depends on the path-orientation-change, it is not aware of the length of the path needed to accumulate the per-cycle $360^{\circ}$ orientation change. At every point on the path, one can trace back along the path to determine a point at $360^{\circ}$ orientation change earlier, and define the equivalent of a 
'previous' cycle at any point on the path. However, if the path involves sections with different curvatures, the path length of this equivalent previous cycle will inevitably be path-position dependent.

To illustrate this, the orientation based method is compared to a constant path length (i.e. equal to the path length of the first full cycle counted) period based method in figure 4, where both methods are used to compute the second order path, i.e. an estimator of the mean path position.
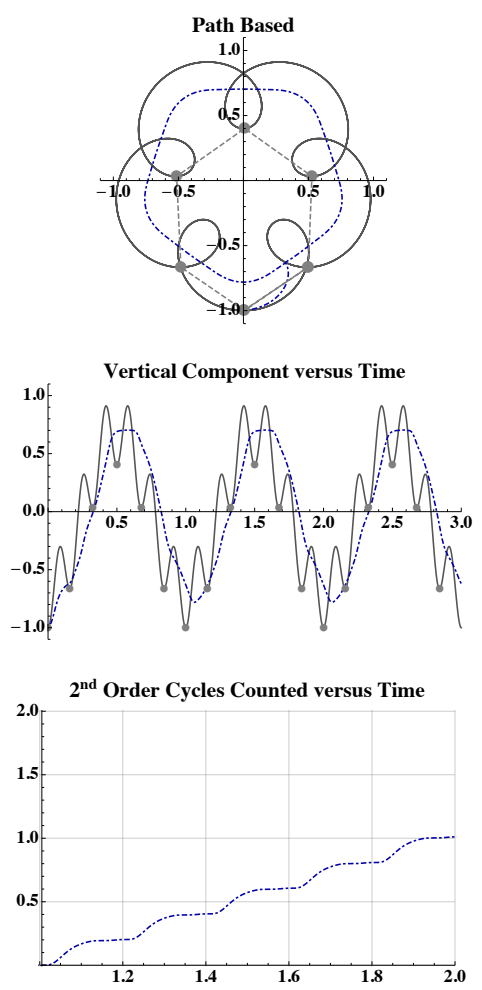
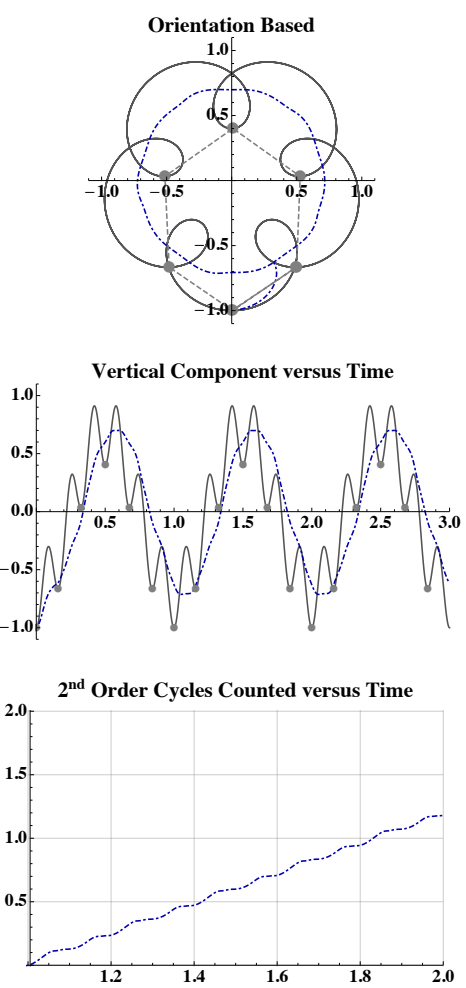

Figure 4. Influence of the way the path mean is computed.

\section{Conclusions and future work}

A new approach is presented to count fatigue cycles. The method is based on a quantification of the orientation change of the path in loading (stress or strain) space. It is completely independent of the criterion used to analyze the cyclic damage accumulation, and is therefore of interest to the analysis of non-proportional, constant and variable amplitude and variable frequency loading cycles. It was illustrated that the method is compatible with classic cycle counting methods when considering uniaxial or proportional multiaxial loading cycles.

The potential of the new cycle counting method is promising but still needs to be established. A first step consists of the tuning and/or development of damage criteria that are compatible with the method, especially addressing the use of its second order cycles to define cyclic amplitude and mean in the definition of a damage criterion. To this purpose extended testing of the method using existing experimental data would be favorable. 


\section{Acknowledgement}

swissnuclear is acknowledged for funding the research leading to this article through the PLiM project within frame contract № 1310 .

\section{References}

[1] ASTM Standard E1049-85 (Reapproved 2017) Standard practices for cycle counting in fatigue analysis, Tech. rep., ASTM International, West Conshohocken, PA (2017)

[2] C.H. Wang, M.W. Brown, A path-independent parameter for fatigue under proportional and non-proportional loading, Fatigue Fract. Eng. M. 16, 1285 (1993)

[3] J.A. Bannantine, D.F. Socie, A variable amplitude multiaxial life prediction method, in: Fatigue under biaxial and multiaxial loading, ESIS 10, edited by K. Kussmaul, D. McDiarmid, D. Socie (London: Mechanical Engineering Publications, 1991), pp. $35-51$

[4] V. Anes, L. Reis, B. Li, M. de Freitas, New cycle counting method for multiaxial fatigue, Int. J. Fatigue 67, 78 (2014)

[5] A. Carpinteri, G. Fortese, C. Ronchei, D. Scorza, S. Vantadori, Spectral fatigue life estimation for non-proportional multiaxial random loading, Theoretical and Applied Fracture Mechanics 83, 67 (2016)

[6] A. Carpinteri, A. Spagnoli, S. Vantadori, A review of multiaxial fatigue criteria for random variable amplitude loads, Fatigue Fract. Engng. Mater. Struct. 40, 1007 (2017)

[7] Y. Wang, L. Susmel, The modified Manson-Coffin curve method to estimate fatigue lifetime under complex constant and variable amplitude multiaxial fatigue loading, Int. J. Fatigue 83, 135 (2016)

[8] J. Mei, P. Dong, S. Kalnaus, Y. Yiang, Z. Wei, A path-dependent fatigue crack propagation model under non- proportional modes I and III loading conditions, Eng. Fract. Mech. 182, 202 (2017)

[9] J. Mei, P. Dong, An equivalent stress parameter for multi-axial fatigue evaluation of welded components including non-proportional loading effects, Int. J. Fatigue 101, 297 (2017)

[10] N. Gates, A. Fatemi, Multiaxial variable amplitude fatigue life analysis including notch effects, Int. J. Fatigue 91, 337 (2016)

[11] M.A. Meggiolaro, J.T.P. de Castro, H. Wu, Non-linear incremental fatigue damage calculation for multiaxial non-proportional histories, Int. J. Fatigue 100, 502 (2017)

[12] T.E. Langlais, J.H. Vogel, T.R. Chase, Multiaxial cycle counting for critical plane methods, Int. J. Fatigue 25, 641 (2003)

[13] K.G.F. Janssens, Proportionally and non-proportionally perturbed fatigue of stainless steel, Int. J. Fatigue 110C, 42 (2018)

[14] Y. Jiang, P. Kurath, Nonproportional cyclic deformation: critical experiments and analytical modeling, Int. J. Plasticity 13, 743 (1997) 